\title{
Application of preoperative three-dimensional model design in radioactive particle implantation for advanced pancreatic cancer
}

This article was published in the following Dove Press journal:

OncoTargets and Therapy

Yang Liu'

Shao-Cheng Lyu

Xian-Qiang Wang ${ }^{3}$

Yan-Bin Wang'

'Department of Hepatobiliary, PLA General Hospital of China, Beijing I00853, People's Republic of China; ${ }^{2}$ Department of Hepatobiliary, Beijing Chaoyang Hospital, Beijing I00020, People's Republic of China; ${ }^{3}$ Department of Pediatric Surgery, PLA General Hospital of China, Beijing I00853, People's Republic of China

Correspondence: Yan-Bin Wang Department of Hepatobiliary, PLA General Hospital of China, No 28 of Fuxing Road, Haidian District, Beijing 100853, People's Republic of China Tel +86 I39 II 6025 I9

Fax +86 10 6818 2255

Email wangyb|5375@163.com
Objective: This study aimed to investigate the application of preoperative three-dimensional model design in radioactive particle implantation for advanced pancreatic cancer, and accordingly analyze the effect of particle implantation in the treatment of advanced pancreatic cancer.

Methods: The clinical data of 63 patients with advanced pancreatic cancer treated with particle implantation from January 2009 to June 2015 in the General Hospital of Chinese PLA were retrospectively analyzed. The implantation design was conducted using the FitMe three-dimensional model reconstruction software for all patients before the operation to explore the significance of preoperative three-dimensional model design in guiding operation. These data were compared with the general data, postoperative recovery, and follow-ups of patients with advanced pancreatic cancer, who underwent conservative treatment at the same time period, in order to explore the effect of particle implantation in the treatment of advanced pancreatic cancer.

Results: In the 63 patients with advanced pancreatic cancer who underwent particle implantation, the average number of implanted particles was 53.4 \pm 18.7 . Gastroparesis occurred in 17 patients and pancreatic fistula occurred in 13 patients after the operation, and no perioperative death occurred.

Follow-up results: In the particle group, the relief rate of abdominal pain was $90.9 \%$, the 1-month, 6-month, 1-year, and 2-year survival rates were $100 \%, 58.7 \%, 22.4 \%$, and $9 \%$, respectively, and median survival time was $10.4 \pm 0.7$ months, which were significantly higher than patients in the control group $(P<0.05)$, especially in patients with stage III pancreatic cancer. Conclusion: For patients with advanced pancreatic cancer who could not undergo radical resection, radioactive particle implantation is an effective treatment, while the use of a preoperative three-dimensional model design for operation planning can maximize the effect of radioactive particles.

Keywords: pancreatic cancer, radiotherapy, ${ }^{125} \mathrm{I}$ particle, 3D model, survival analysis

\section{Introduction}

Pancreatic cancer is a malignant tumor with extremely high malignancy in the digestive tract, which has very poor prognosis. It ranks fourth among the causes of global cancer deaths. ${ }^{1,2}$ Surgical radical resection is the only cure for pancreatic cancer. However, only $5 \%-20 \%$ of the patients can receive radical resection. ${ }^{3}$ A 20 -year national survey of pancreatic cancer in Japan revealed that the 5-year survival rate was $13.4 \%$ for patients undergoing surgical resection, and $0.8 \%$ and $0 \%$ for patients undergoing palliative and nonoperative treatments, respectively. ${ }^{4}$ For patients with pancreatic cancer who could not undergo radical resection, chemotherapy and radiotherapy are the only strategies that could prolong survival time. 
Radiotherapy is a mean for the comprehensive treatment of pancreatic cancer. ${ }^{5}$ However, in conventional external radiotherapy, the rays are attenuated by the skin, muscle, and visceral layers, preventing the treatment from achieving good results, and affecting the overall prognosis of patients. ${ }^{6}$ Therefore, determining how to effectively focus radiation on the tumor and reduce damage to normal tissues and cells has been the focus of radiotherapy research. In 1972, Whitmore et $\mathrm{al}^{7}$ was the first to apply ${ }^{125} \mathrm{I}$ to treat prostate cancer and achieved success. From then on, the implantation of particles began to be applied in the treatment of tumors in various parts of the human body, which achieved good results.

However, there is no unified conclusion about how to distribute the particles evenly into the tumor. ${ }^{8,9}$ With the development of medical imaging technology, three-dimensional (3D) imaging technique of tumors and its surrounding tissues has been realized in clinical practice. If 3D imaging technology could be introduced into the particle implantation technology, it can provide a more accurate guidance for the path, number, and direction of particle implantation before surgery. This would make particle implantation technology more planned and accurate and allow the radiation dose distribution to be the highest in the tumor area and relatively low in the surrounding areas, accordingly achieving the best treatment effect. ${ }^{10}$ In the present study, FitMe 3D model reconstruction software was applied to ${ }^{125} \mathrm{I}$ particle implantation in patients with advanced pancreatic cancer. The preoperative 3D model design was used to guide the implantation of particles in the operation, evaluate the effect of particle implantation and the perioperative condition of patients, and ultimately evaluate the prognosis of patients.

\section{Data and methods}

This study was conducted in accordance with the Declaration of Helsinki. This study was conducted with approval from the Ethics Committee of PLA General Hospital of China. Written informed consent was obtained from all participants.

\section{General clinical data}

A total of 63 patients with advanced pancreatic cancer treated with ${ }^{125}$ I implantation from January 2009 to June 2015 in the General Hospital of Chinese PLA were included into this study. Among these patients, 38 patients were male and 25 patients were female. The age of these patients ranged from 36 to 87 years, with an average age of $57.9 \pm 12.2$ years. Primary symptoms included the following: abdominal pain (33 patients), jaundice (16 patients), and digestive tract symptoms (10 patients). Four patients were diagnosed during the physical examination. Tumor locations were as follows: the head of the pancreas (42 patients) and the tail of the pancreas (42 patients). With regard to preoperative TNM staging, 52 patients were at stage III and 11 patients were at stage IV.

Inclusion criteria were as follows: 1) patients clinically diagnosed with pancreatic cancer; 2) patients who were at TNM stage III or IV of pancreatic cancer, and could not undergo radical surgery; 3) patients with pancreatic cancer confirmed by pathology during or after the operation; 4) patients with a life expectancy of $\geq 3$ months; 5) patients or their families agreed to implement particle implantation; and 6) patients who could tolerate surgery.

\section{Radiation source}

The used ${ }^{125} \mathrm{I}$ particles were provided by Beijing Zhibo BioMedical Technology Co., Ltd. (Beijing, People's Republic of China), which were cylindrical particles with a diameter of $0.8 \mathrm{~mm}$ and a length of $4.5 \mathrm{~mm}$. The mean photon energy was $27-35 \mathrm{keV}$, the intertissue penetration was $1.7 \mathrm{~cm}$, and the physical half-life was 59.6 days.

\section{Three-dimensional simulated implantation design}

\section{Acquisition and processing of data}

Enhanced computed tomography (CT) scan was performed before surgery. CT images were acquired using the 256slice spiral CT (Philips Brilliance iCT, Philips, Amsterdam, the Netherlands), and the thickness of slice was $1.25 \mathrm{~mm}$. The images were stored in DICOM format and imported in to the FitMe 3D reconstruction software system (a domestic software, developed by Feite United Graph; and the author was authorized to use it) to perform the $3 \mathrm{D}$ reconstruction of the tumor and its peripheral blood vessels. The reconstructed data were saved in AVI video format and JPEG picture format, respectively, to facilitate the observation on the processing of the surgical design in the overall view under dynamic conditions and in the local view under static conditions.

\section{Calculation of parameters}

Using this software system, the volume of the pancreatic tumor in 63 patients (volume unit: $\mathrm{mL}$ ) was measured in real time, and the specific location of the tumor was depicted. Then, from various angles, such as the ventral side (A), dorsal side $(\mathrm{P})$, right side $(\mathrm{R})$, left side $(\mathrm{L})$, head side $(\mathrm{H})$, and foot side $(\mathrm{F})$, the relationship between the tumor and peripheral vessels was analyzed to evaluate the relationship and distance between the tumor and blood vessels (distance unit: mm, Figure 1). 

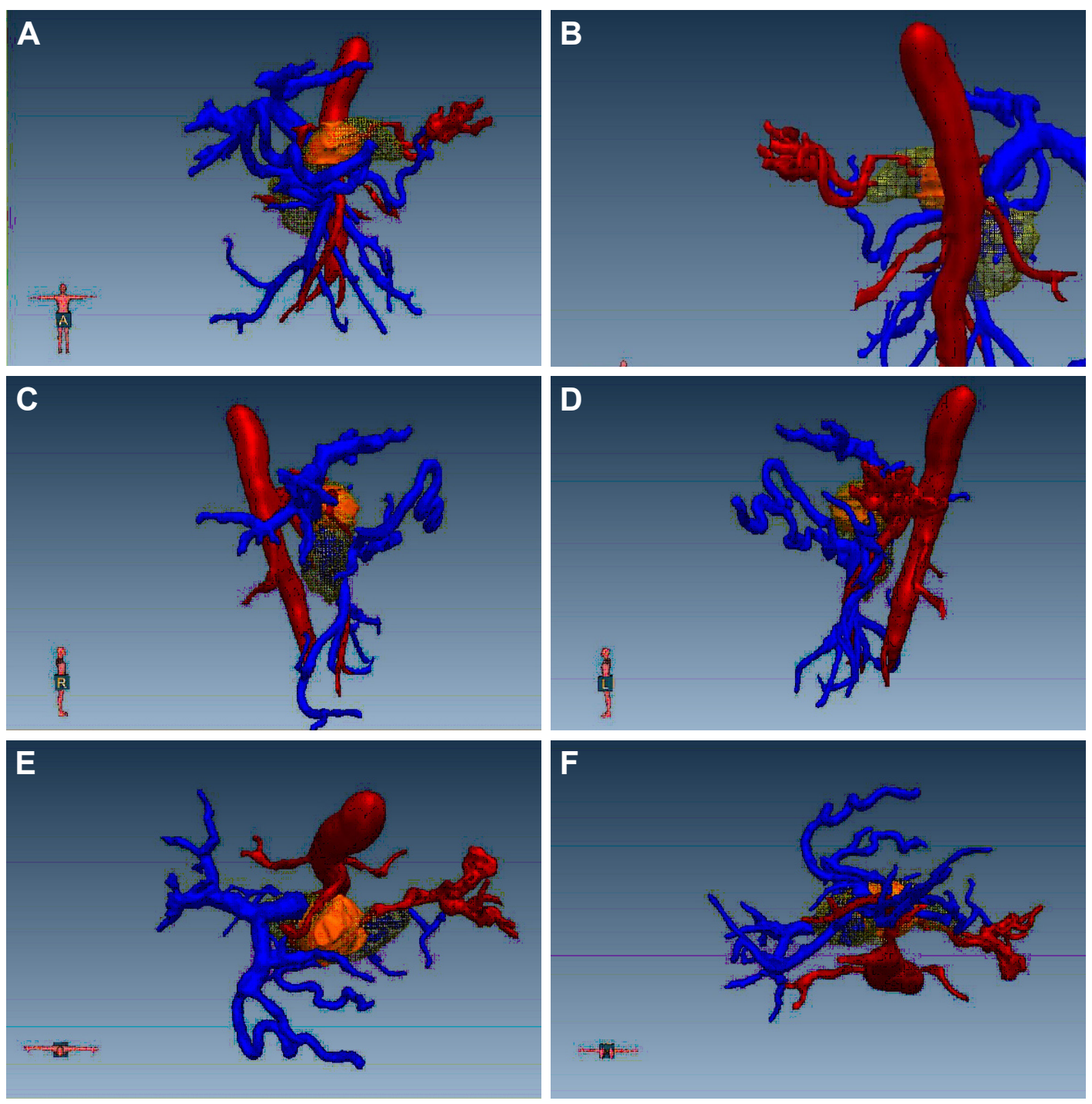

Figure I Schematic diagram of local analysis.

Notes: (A) Ventral view (A); (B) dorsal view (P); (C) right view (R); (D) left view (L); (E) head view (H); (F) foot side view (F).

\section{Design of virtual surgery of particle implantation}

The spatial distribution of particles conformed to the principles of the Paris system: 1) the particles were arranged in parallel lines in space; 2) the particles are equidistantly arranged $(15-20 \mathrm{~mm}) ; 3)$ the particles in a line have a same kerma rate; 4) the spatial distribution of the particles in a cross-section is a square or an equilateral triangle; 5) on the plane of the space center of the particles, the mean value of the sum of the dose rates in the center hole is the basic dose of the particles; and 6) the locations of the particles embedded in the tumor are more than $0.5 \mathrm{~cm}$ below the tumor surface. According to these basic principles, a supplementary principle was put forward: in implantation, the particles planned to be implanted in the most peripheral areas are first implanted, namely, the principle of "edge encircling the center." According to these principles, 3D-aided design was carried out, and the direction and position of the particle implantation were virtually designed to plan the spatial location of each implant point and implant needle path, and calculate the number of particles implanted (since the specific drawing process was complex, only the completed particle implantation model is shown). The darker inner layer was the tumor, and the outer layer was the radiation volume of the particles, that is, the radioactive range of the implanted particles completely covers the entire tumor. This is shown in Figures 2A-D and 3. On this basis, the particles were ordered, and usually ten more particles were ordered for reserves, in order to prepare for the occurrence of "blind spot" and "cold spot" in the operation. 

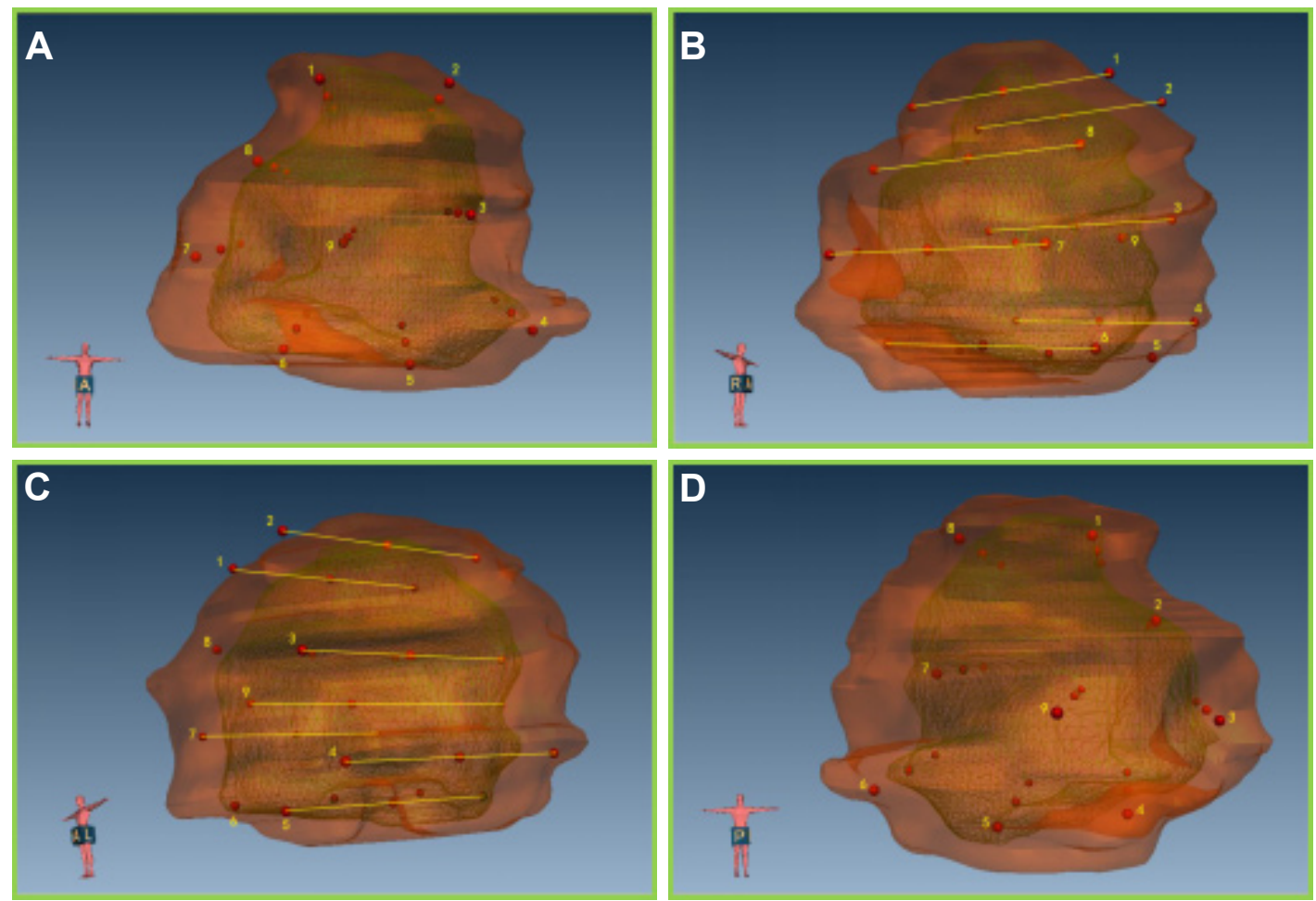

Figure 2 The distribution of implanted particles from all angles, which involve nine implantation sites.

Notes: The yellow straight lines refer to needle paths. The number of implanted particles in the plan (27) was calculated, and the model of the particle implantation was established. (A) Nine implantation sites showed from the view of facies ventralis; (B) needle paths and implantation sites showed from the view of the right side; (C) needle paths and implantation sites showed from the view of the left side; (D) nine implantation sites showed from the view of the back.

\section{Comparison between groups}

A total of 89 patients with advanced pancreatic cancer treated with palliative surgery in our hospital during the same time period were included. The comparison of general data between these two groups of patients is presented in Table 1. Differences in general data between these two groups were not statistically significant $(P>0.05)$. Hence, these two groups of patients were comparable.

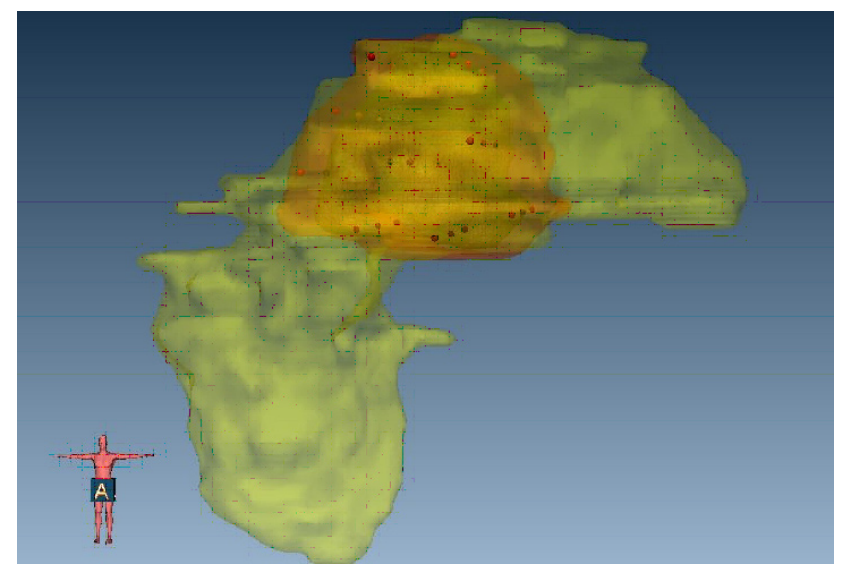

Figure 3 Holistic view of the 3D design of the particle implantation.

\section{Follow-ups and statistical analysis}

The endpoint of follow-ups for these patients was December 2015, and these patients were followed up by outpatient review and telephone call. The normally distributed data were expressed as mean $\pm \mathrm{SD}$, and data in nonnormal distribution were expressed as the median (interquartile range). Count data were compared between these two groups using independent $t$-test or rank sum test, and measurement data were evaluated using chi-squared test. The survival curve was calculated using the Kaplan-Meier method, and the survival curves between groups were compared using the log-rank test. $P<0.05$ was considered statistically significant. Data were statistically analyzed using statistical software SPSS 12.0 (SPSS Inc., Chicago, IL, USA).

\section{Results}

\section{Implantation of the ${ }^{125}$ particle}

Based on the preoperative 3D design planning, the patients in the particle group were evenly implanted with particles according to the size and spatial distribution of the tumor. Ultrasound was used to evade vessels and dilate the pancreatic duct during the operation. An 18-G PTB needle (provided by Beijing Zhibo Bio-Medical Technology Co., Ltd.) was used 
Table I Comparison of general data between groups

\begin{tabular}{l|l|l|l}
\hline Items & Particle group (n=63) & Control group (n=89) & -value \\
\hline Gender (male/female) & $38 / 25$ & $64 / 25$ & 0.1340 \\
Age (years old) & $57.9 \pm 12.2$ & $56.4 \pm 11.3$ & 0.4280 \\
Tumor locations (head/tail) & $42 / 21$ & $64 / 25$ & 0.4882 \\
\hline TNM stage & & & \\
\hline III stage & 52 & 65 & 0.1703 \\
IV stage & $\mathrm{II}$ & 24 & \\
\hline
\end{tabular}

to puncture from the entry point to the bottom of the tumor, and the inner core was withdrawn. Then, it was observed to determine whether the blood or pancreatic juice outflowed from the needle core. If not, the implantation was started: at approximately $5 \mathrm{~mm}$ from the edge of the tumor, a particle was implanted at the bottom of the tumor, the needle was retreated by $1 \mathrm{~cm}$, a particle was implanted again, and the needle was retreated by $1 \mathrm{~cm}$ again; then, a particle was implanted again, implantation was repeatedly carried out according to this method until approximately $5 \mathrm{~mm}$ away from the top edge of the tumor. The interval between the puncture and implantation sites was $1 \mathrm{~cm}$. Resow was conducted at special positions and deep sites, namely, "blind spots" and "cold spots," in order to ensure that the particles covered the tumor. In order to prevent the occurrence of delayed oozing of blood or oozing of fluid in the puncture sites, an "8-shaped" suture was performed with 5-0 Prolene suture at each puncture point. The number of practically implanted particles was often more than the particles in the plan, because there was a certain error between the results of direct vision and imaging, which is called the "blind spot" and "cold spot."

The patients in the control group only underwent palliative surgery (laparotomy or bypass). All patients were routinely treated with supportive treatment, such as nutritional support, anti-inflammatories, and acid suppression therapy. The urinary catheter was removed from all patients on postoperative day 1 , and the gastric tube was removed based on the recovery of the patient (already exhaust, bowel sounds and gastric juice $<300 \mathrm{~mL} / \mathrm{d}$, no bile or blood-like materials), and the drainage tube was removed according to the characteristics of the drainage fluid $(<20 \mathrm{~mL} / \mathrm{d}$, no pancreatic fistula, infection and bleeding). Patients were arranged for discharge according to postoperative recovery.

\section{Perioperative conditions}

In the present study, no perioperative death occurred. In the particle group, the maximal diameters of the tumors ranged within 3-10 cm, and the average maximal diameter of the tumor was $5.2 \pm 1.7 \mathrm{~cm}$. The number of particles implanted

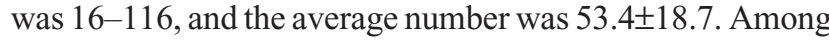
these patients, 7 patients underwent gastroenteroanastomosis, 14 patients underwent bile duct anastomosis, 20 patients simultaneously underwent bile duct anastomosis and gastroenteroanastomosis, and 22 patients underwent only particle implantation. In the particle group, gastroparesis occurred in 17 patients after the operation, biliary fistula occurred in 1 patient, abdominal infection occurred in 1 patient, and pancreatic fistula occurred in 13 patients. Twelve patients were at grade $\mathrm{A}$ and 1 patient was at grade $\mathrm{B}$. One patient developed abdominal bleeding, which occurred on postoperative day 12 ; this was cured after conservative treatment. The comparison of perioperative conditions between these two groups is presented in Table 2. Particle implantation increased the operation duration and the incidence of postoperative gastroparesis and pancreatic fistula, but it did not increase the intraoperative bleeding volume and length of hospital stay.

\section{Follow-ups}

The endpoint of follow-ups for patients was December 2015, and the duration of follow-ups ranged within 1-29 months, with a median duration of follow-up of 6 months. The review results of some patients in the particle groups are shown in Figure 4. The overall follow-up rate was $92.8 \%(141 / 152)$. The follow-up rate was $92.1 \%(58 / 63)$ in the particle group and $93.3 \%(83 / 89)$ in the control group. Among the 33 patients who complained of abdominal pain upon visiting the doctor in the particle group, three patients continued to have abdominal pain during the follow-ups, and the relief rate of abdominal pain was $90.9 \%$. Among all these patients, 32 patients were treated with adjuvant systemic chemotherapy after discharge, and 3 patients were treated with local radiotherapy. The comparison of the postoperative basic data between these two groups is shown in Table 3 .

In the present study, the 1-month, 6-month, 1-year, and 2-year survival rates of all patients who were followed up were $98.6 \%, 46.8 \%, 13.8 \%$, and $4.9 \%$, respectively, and the median survival time was $8.0 \pm 0.3$ months. The 1 -month, 6-month, 1-year, and 2-year survival rates of patients in 
Table 2 Comparison of perioperative conditions between two groups

\begin{tabular}{|c|c|c|c|}
\hline Items & Particle group $(n=63)$ & Control group $(n=89)$ & $P$-value \\
\hline Tumor diameter $(\mathrm{cm})$ & $5.2 \pm 1.7$ & $5.4 \pm 2.5$ & 0.6950 \\
\hline Operation time (hours) & $4.7 \pm 1.4$ & $3.3 \pm 1.4$ & $0.0000^{\mathrm{a}}$ \\
\hline Bleeding volume during operation $(\mathrm{mL})$ & $190 \pm 117$ & $|64 \pm| 4 \mid$ & 0.2232 \\
\hline Postoperative hospital stay (days) & $13.4 \pm 4.1$ & $12.1 \pm 6.5$ & 0.1455 \\
\hline \multicolumn{4}{|l|}{ Operation method } \\
\hline Gastrointestinal + biliary-intestinal & 20 & 33 & 0.4967 \\
\hline Gastrointestinal & 7 & 4 & 0.2175 \\
\hline Biliary-intestinal & 14 & 31 & 0.0934 \\
\hline \multicolumn{4}{|l|}{ Postoperative complications } \\
\hline Pancreatic fistula & 13 & 3 & $0.0006^{\mathrm{a}}$ \\
\hline Gastroparesis & 17 & 3 & $0.0000^{\mathrm{a}}$ \\
\hline Bleeding & 1 & 2 & 0.7613 \\
\hline Intra-abdominal infection & I & 2 & 0.7613 \\
\hline Biliary fistulas & I & 3 & 0.8710 \\
\hline
\end{tabular}

Note: ${ }^{a} \mathrm{P}<0.05$.

the particle group, who were followed up, were $100 \%$, $58.7 \%, 22.4 \%$, and $9 \%$, respectively, and the median survival time was $10.4 \pm 0.7$ months. The 1-month, 6-month, 1 -year, and 2-year survival rates of patients in the control group, who were followed up, were $97.6 \%, 38.6 \%, 8.4 \%$, and $0 \%$, respectively, and the median survival time was $6.8 \pm 0.2$ months. The comparison of survival curves in the two groups is shown in Figure 5. The 1-month, 6-month, 1-year, and 2-year survival rates were significantly higher in the particle group than in the control group. Log-rank test revealed that $\mathrm{QPH}=7.949$ and $P=0.0048$, and the difference was statistically significant.

Stratified analysis according to TNM staging of tumor: for patients with stage III pancreatic cancer, the 1-month, 6-month, 1-year, and 2-year survival rates were 100\%, 57.6\%, $20.2 \%$, and $10.8 \%$ in the particle group, respectively, and
$100 \%, 40.7 \%, 10 \%$, and $3.3 \%$ in the control group, respectively; mean survival time was $10.6 \pm 0.9$ and $7.4 \pm 0.2$ months in the particle group and control group, respectively. The comparison of survival curves in these two groups is shown in Figure 6: the 1-month, 6-month, and 1-year survival rates were significantly higher in the particle group than in the control group. Log-rank test revealed that $\mathrm{QPH}=4.446$ and $P=0.0350$, and the difference was statistically significant. For patients with stage IV pancreatic cancer, the 1-month, 6-month, 1-year, and 2-year survival rates were $100 \%, 63.6 \%$, $31.8 \%$, and $0 \%$ in the particle group, respectively, and $91.7 \%$, $33.3 \%, 4.8 \%$, and $0 \%$ in the control group, respectively; the mean survival time was $9.5 \pm 1.6$ and $5.8 \pm 0.4$ months in the particle group and control group, respectively. The comparison of survival curves in these two groups is shown in Figure 7: the 1-month, 6-month, and 1-year survival rates
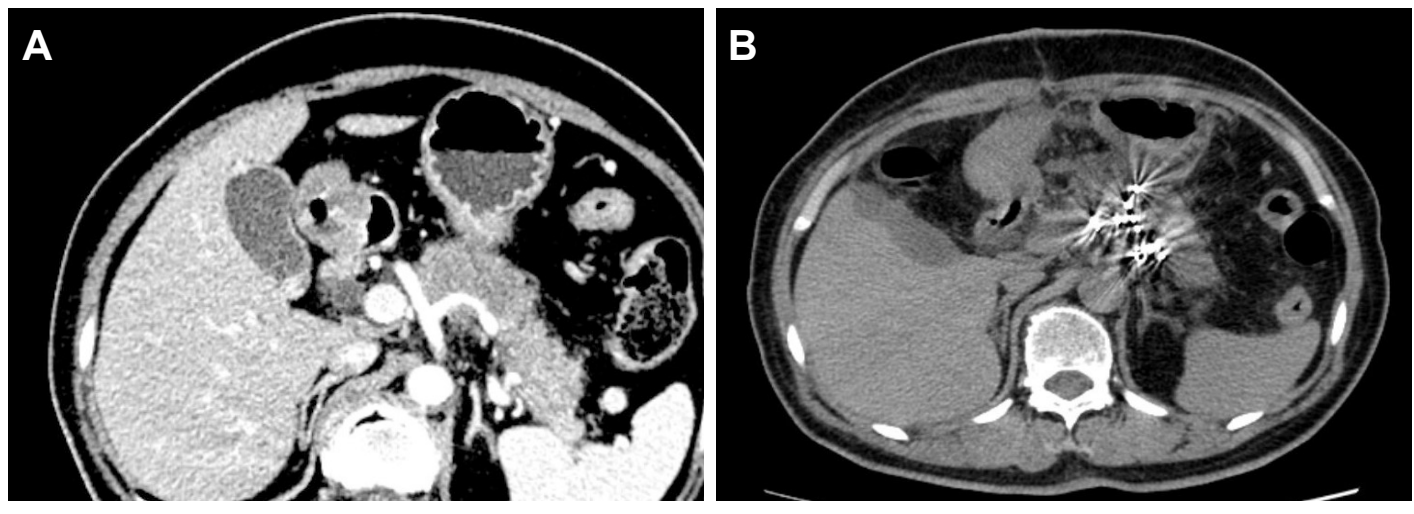

Figure 4 The patient is a 62-year-old female, who had carcinoma in the body and tail of the pancreas.

Notes: The subject was preoperatively evaluated to be at stage III. This figure shows the situation at I month after the implantation of particles. (A) The CT shows that tumor invaded the splenic artery; (B) the tumor disappeared I month after the implantation.

Abbreviation: CT, computed tomography. 
Table 3 Comparison of the postoperative basic data between these two groups

\begin{tabular}{|l|l|l|l|}
\hline Items & $\begin{array}{l}\text { Particle } \\
\text { group (n=63) }\end{array}$ & $\begin{array}{l}\text { Control } \\
\text { group (n=89) }\end{array}$ & P-value \\
\hline $\begin{array}{l}\text { Abdominal pain relief/ } \\
\text { nonrelief (cases) }\end{array}$ & $30 / 3$ & $10 / 43$ & $0.0000^{*}$ \\
\hline $\begin{array}{l}\text { Postoperative } \\
\text { chemotherapy (cases) }\end{array}$ & 32 & 57 & 0.1020 \\
\hline $\begin{array}{l}\text { Postoperative local } \\
\text { radiotherapy (cases) }\end{array}$ & 3 & 37 & $0.0000^{*}$ \\
\hline
\end{tabular}

Note: $* P<0.05$.

were significantly higher in the particle group than in the control group. Log-rank test revealed that $\mathrm{QPH}=2.845$ and $P=0.0916$, and the difference was not statistically significant.

The reason may be related to the small number of patients with stage IV in the particle group. Further research is needed to confirm these results.

\section{Discussion}

Particle implantation has been a new radiotherapy method for malignant tumors in recent years. When radioactive particles are implanted into the human body, these can produce continuous radiation rays to destroy the tumor. ${ }^{11}$ Radioactive particle implantation is a safe and minimally invasive local radiotherapy method, and it has incomparable advantages compared to external radiotherapy. ${ }^{12,13}$

Particle implantation is a conformal radiotherapy. It not only requires strict radiation dosimetry for assurance, but also requires strict planning to prevent side injuries. Therefore, particle implantation therapy must be guided by dosimetry and requires the development of a reasonable treatment plan. ${ }^{14}$ The ideal dose distribution is that the deep area of the tumor receives a high dose, while the surrounding normal tissue receives a low dose, and the dose distribution is uniform within the tumor region. At the advent of particle implantation, due to the lack of computer-aided technology, particle implantation involves a certain degree of blindness, and consequently results in a poor curative effect. With the clinical application of the computer-aided 3D therapeutic planning system, particle implantation has been gradually improved. The application of the computer-aided 3D therapeutic planning system can significantly improve accurate localization in particle implantation and achieve a highly individualized conformal dose distribution, thereby improving the effect of particle therapy, and reducing the incidence of postoperative complications. ${ }^{15}$ The General Hospital of Chinese PLA introduced the FitMe 3D reconstruction system to carry out preoperative auxiliary surgical planning for particle implantation. The 3D reconstruction system automatically connects the model and obtains the exterior orientation elements and camera parameters by close-shot aerotriangulation, thereby automatically forming point clouds of 3D coordinate points of the object area, in order to establish a high-precision human organ model ${ }^{16}$ and guide the 3D distribution of implanted particles in the tumor and its surrounding area. Furthermore, this would allow the radiation emitted by the implanted particles to effectively cover the tumor and subclinical regions in the tumor margins, destroying tumor cells to the maximum extent. Under the guidance of the 3D therapeutic planning system, unnecessary irradiation and damage caused by excessive local dose are avoided, and reimplantation for cold zones after the operation due to too low local dose is avoided. ${ }^{17}$

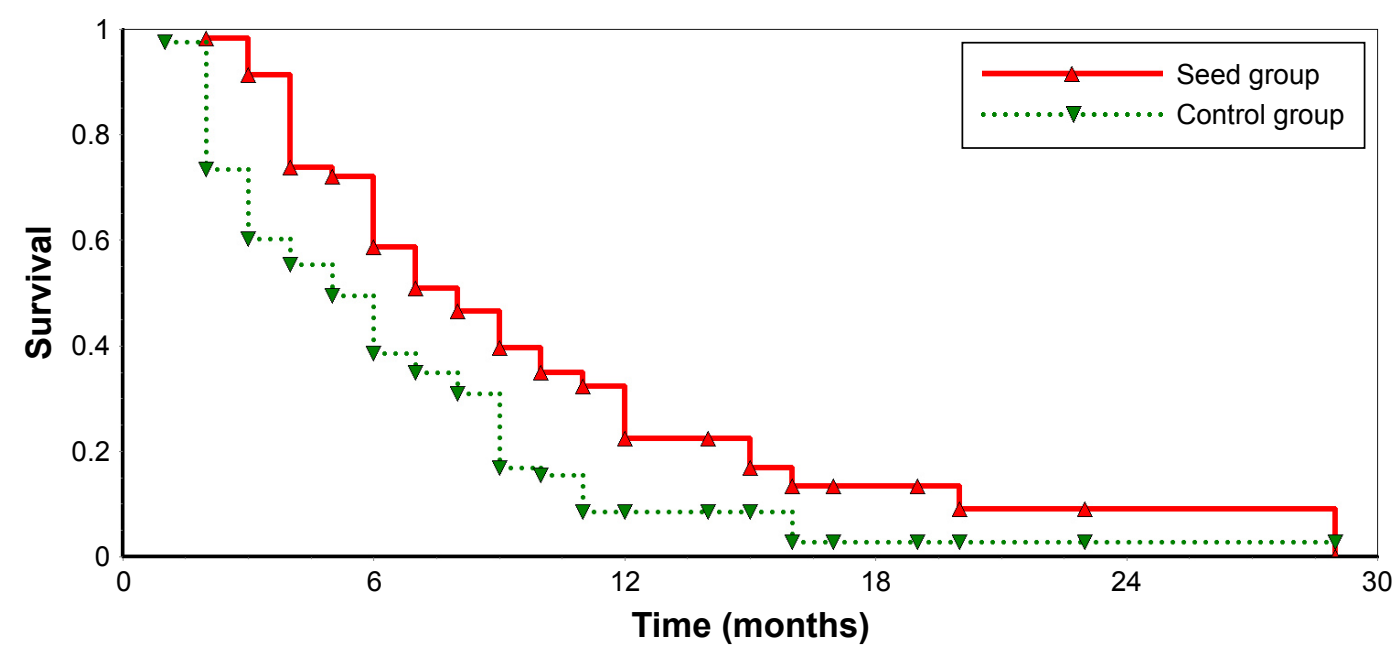

Figure 5 The overall survival curves of patients in the two groups who were followed up. 


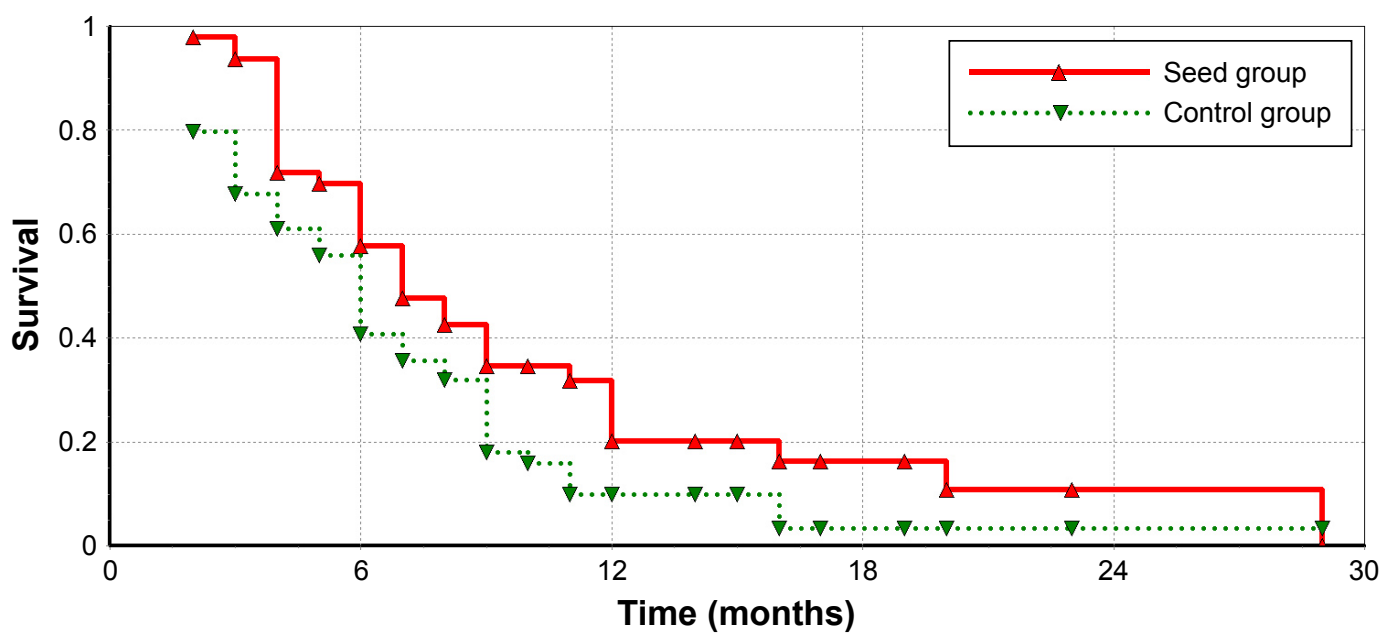

Figure 6 Comparison of survival curves between the two groups of patients with stage III tumors who were followed up.

At present, particle implantation has been used in the clinical treatment of various malignant tumors, and good clinical efficacy was achieved. ${ }^{18}$ After ${ }^{125}$ I radioactive particles were implanted into the tumor, these could produce continuous $\mathrm{X}$-rays and $\gamma$-rays to destroy the tumor. ${ }^{19}$ Studies at home and abroad have revealed that the survival time of patients with advanced pancreatic cancer is approximately 6 months, ${ }^{20,21}$ while in the present study, the 1-month, 6-month, 1-year, and 2 -year survival rates were $100 \%, 58.7 \%, 22.4 \%$, and $9 \%$, respectively, and the median survival time was $10.4 \pm 0.7$ months. Furthermore, the present study also revealed that particle implantation increased the risk of postoperative pancreatic fistula and gastroparesis, but this did not increase perioperative mortality rate. Therefore, particle implantation is relatively safe and feasible for patients with advanced pancreatic cancer. In addition, through radioactive rays, the posterior pancreatic nerve can also be inactivated, and it can also reduce the compression of tumors on surrounding tissues and the pancreatic duct, and the stimulation of tumor to the pancreatic capsule, achieving an analgesic effect. ${ }^{22}$ Wang et $\mathrm{al}^{23}$ reported a study that involved 13 pancreatic cancer patients with local progression, who could not undergo surgical resection after particle implantation. The relief rate was $100 \%$, and the effective rate was $69.2 \%$. In the present study, the relief rate of abdominal pain was $90.9 \%$ in the particle group.

The ${ }^{125}$ I particle implantation can significantly prolong the survival time of patients with advanced pancreatic cancer, and is an effective treatment for patients with advanced pancreatic cancer. ${ }^{24}$ The application of a preoperative 3D

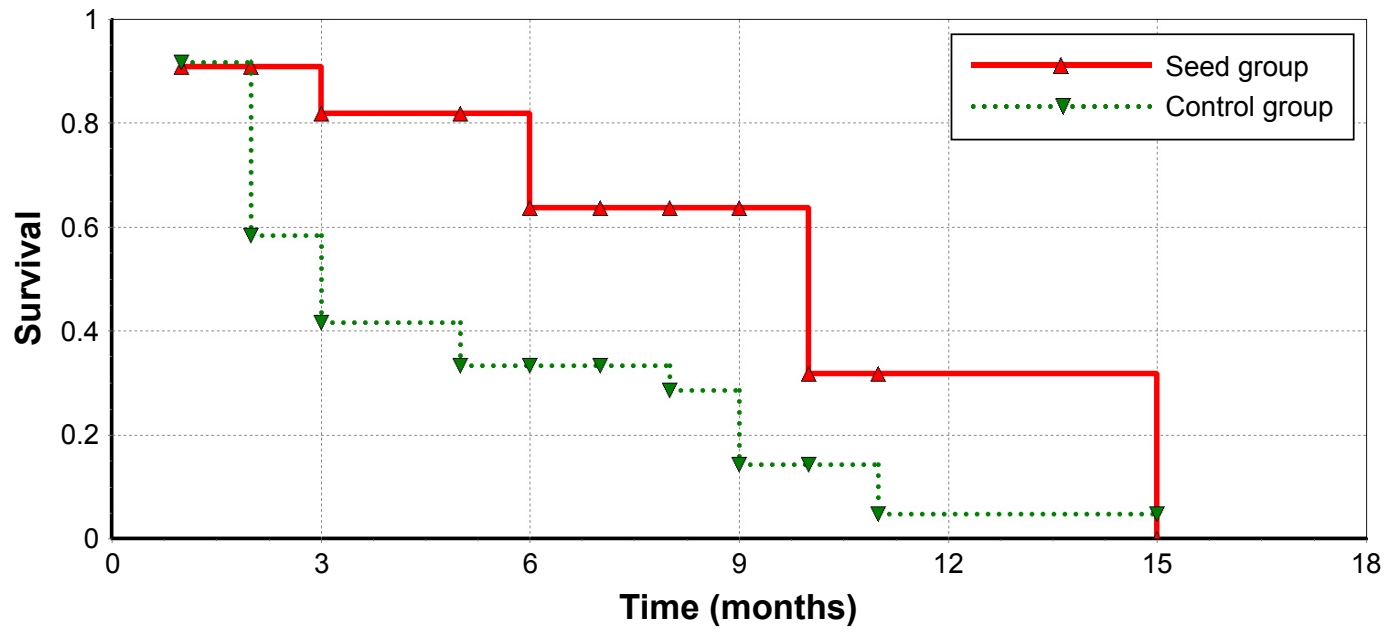

Figure 7 Comparison of survival curves between the two groups of patients with stage IV tumors who were followed up. 
model for auxiliary planning can maximize the effect of radioactive particles and reduce the complications of particle implantation.

\section{Conclusion}

For patients with advanced pancreatic cancer who could not undergo radical resection, radioactive particle implantation is an effective treatment, while the use of a preoperative three-dimensional model design for operation planning can maximize the effect of radioactive particles.

\section{Disclosure}

The authors report no conflicts of interest in this work.

\section{References}

1. Cooperman AM. Pancreatic cancer: the bigger picture. Surg Clin North Am. 2001;81(3):557-574.

2. Christians K, Evans DB. Pancreaticoduodenectomy and vascular resection: persistent controversy and current recommendations. Ann Surg Oncol. 2009;16(4):789-791.

3. Kelleher FC. Hedgehog signaling and therapeutics in pancreatic cancer. Carcinogenesis. 2011;32(4):445-451.

4. Matsuno S, Egawa S, Fukuyama S, et al. Pancreatic Cancer Registry in Japan: 20 years of experience. Pancreas. 2004;28(3):219-230.

5. Jemal A, Siegel R, Xu J, Ward E. Cancer statistics, 2010. CA Cancer J Clin. 2010;60(5):277-300.

6. Baron TH, Kozarek RA. Preoperative biliary stents in pancreatic cancer - proceed with caution. N Engl J Med. 2010;362(2):170-172.

7. Whitmore WF, Hilaris B, Grabstald H, Batata M. Implantation of $125 \mathrm{I}$ in prostatic cancer. Surg Clin North Am. 1974;54(4):887-895.

8. Wang H, Wang J, Jiang Y, et al. The investigation of $125 \mathrm{I}$ seed implantation as a salvage modality for unresectable pancreatic carcinoma. J Exp Clin Cancer Res. 2013;32:106.

9. Wang J, Jiang Y, Li J, Tian S, Ran W, Xiu D. Intraoperative ultrasoundguided iodine-125 seed implantation for unresectable pancreatic carcinoma. J Exp Clin Cancer Res. 2009;28:88.

10. Kelleher FC. Hedgehog signaling and therapeutics in pancreatic cancer. Carcinogenesis. 2011;32(4):445-451.
11. Zhang Z, Wang J, Shen B, Peng C, Zheng M. The ABCC4 gene is a promising target for pancreatic cancer therapy. Gene. 2012;491(2): 194-199.

12. Zhongmin W, Yu L, Fenju L, Kemin C, Gang H. Clinical efficacy of CT-guided iodine-125 seed implantation therapy in patients with advanced pancreatic cancer. Eur Radiol. 2010;20(7):1786-1791.

13. Vincent A, Herman J, Schulick R, Hruban RH, Goggins M. Pancreatic cancer. Lancet. 2011;378(9791):607-620.

14. Franca CA, Vieira SL, Carvalho AC, Bernabe AJ, Penna AB. Radioactive seed migration after prostate brachytherapy with iodine-125 using loose seeds versus stranded seeds. Int Braz J Urol. 2009;35(5): 573-580.

15. Mei QL, Liu PC, Yang JY, Du RM, Chen ZZ. Safety evaluation of iodine-125 seed implantation in rabbit liver tissue. Nan Fang Yi Ke Da Хие Хие Вао. 2007;27(5):675-678.

16. Callery MP, Chang KJ, Fishman EK, Talamonti MS, William Traverso L, Linehan DC. Pretreatment assessment of resectable and borderline resectable pancreatic cancer: expert consensus statement. Ann Surg Oncol. 2009;16(7):1727-1733.

17. Ko AH, Venook AP, Bergsland EK, et al. A phase II study of bevacizumab plus erlotinib for gemcitabine-refractory metastatic pancreatic cancer. Cancer Chemother Pharmacol. 2010;66(6):1051-1057.

18. Zhang $\mathrm{L}, \mathrm{Mu} \mathrm{W}, \mathrm{Hu} \mathrm{CF}$, Huang XQ. Treatment of portal vein tumor thrombus using ${ }^{12} 5$ Iodine seed implantation brachytherapy. World $J$ Gastroenterol. 2010;16(38):4876-4879.

19. Kim HS, Yi SY, Jun HJ, et al. Definitive chemoradiation therapy with capecitabine in locally advanced pancreatic cancer. Anticancer Drugs. 2010;21(1):107-112.

20. Mamon HJ, Niedzwiecki D, Hollis D, et al. A phase 2 trial of gemcitabine, 5 -fluorouracil, and radiation therapy in locally advanced nonmetastatic pancreatic adenocarcinoma: cancer and Leukemia Group B (CALGB) 80003. Cancer. 2011;117(12):2620-2628.

21. Zhang FJ, Wu PH, Zhao M, et al. CT guided radioactive seed $125 \mathrm{I}$ implantation in treatment of pancreatic cancer. Zhonghua Yi Xue Za Zhi. 2006;86(4):223-227. Chinese.

22. Yamada S, Fujii T, Sugimoto H, et al. Aggressive surgery for borderline resectable pancreatic cancer: evaluation of National Comprehensive Cancer Network guidelines. Pancreas. 2013;42(6):1004-1010.

23. Wang JJ, Yuan HS, Li JN, Jiang YL, Tian SQ, Yang RJ. CT-guided radioactive seed implantation for recurrent rectal carcinoma after multiple therapy. Med Oncol. 2010;27(2):421-429.

24. Zhang JQ, Huang XQ, Zhang J, et al. CT guided radioactive seed (125) I implantation in treating multiple bone metastasis. Zhonghua Yi Xue Za Zhi. 2008;88(39):2739-2742.
OncoTargets and Therapy

\section{Publish your work in this journal}

OncoTargets and Therapy is an international, peer-reviewed, open access journal focusing on the pathological basis of all cancers, potential targets for therapy and treatment protocols employed to improve the management of cancer patients. The journal also focuses on the impact of management programs and new therapeutic agents and protocols on

\section{Dovepress}

patient perspectives such as quality of life, adherence and satisfaction. The manuscript management system is completely online and includes a very quick and fair peer-review system, which is all easy to use. Visit http://www.dovepress.com/testimonials.php to read real quotes from published authors. 